\title{
Narrow Band Imaging Can Be Used for Anastomotic Assessment
}

\author{
Ahmed Z. Kaleem ${ }^{1,2 *}$, Nabish Naheed ${ }^{1,2}$, Syed M. Ahmad ${ }^{1,2}$ \\ ${ }^{1}$ Department of Colorectal Surgery, Northern Lincolnshire and Goole Hospital, Scunthorpe, UK \\ ${ }^{2}$ NHS Foundation Trust, Scunthorpe General Hospital, Scunthorpe, UK \\ Email: *Ahmed.kaleem@nhs.net
}

Received 2 September 2015; accepted 26 February 2016; published 29 February 2016

Copyright (C) 2016 by authors and Scientific Research Publishing Inc.

This work is licensed under the Creative Commons Attribution International License (CC BY). http://creativecommons.org/licenses/by/4.0/

(c) (i) Open Access

\begin{abstract}
Background: Anastomotic leak is the most dreaded complication and it accounts for one third of post-operative deaths in colorectal surgery. There is strong need of research in this field in order to reduce anastomotic leak rate. If there was a test available to signify probability of leak at the time of surgery, it would potentially avoid a leak at a later date. Aim: We propose that intra operative use of narrow band imaging colonoscopy in left sided colonic anastomosis can predict and detect anastomosis related problems, problems i.e. vascularity, anastomotic lumen patency and rigidness of anastomosis which in turn lead to correction at the time of surgery resulting in significant decrease in anastomotic leak rate associated with morbidity and mortality. Methodology: A pilot, single blind, prospective, randomised controlled trial. Aim is to divide patient in 2 groups, containing 15 patients in each group. One group will be assessed by intra-operative NBI colonoscopy and other with standard technique. Analysis: This will be performed using SPSS with the help of statistician. Significance: This area of research is particularly important because it improves survival by reducing morbidity and mortality associated with anastomotic leak. If a leak is avoided then it does not only mean early recovery but it also saves NHS in terms of ICU care, NHS bed, inpatient nursing care and home help, unfortunately all required if leak is to occur. As a result, this research will have better financial implication with significantly improve patient care.
\end{abstract}

\section{Keywords}

Anastomotic Leak, Colorectal Surgery, Anastomotic Assessment

\section{Background}

Learning is a process which leads to specialist development. It is a responsibility of every healthcare individual

*Corresponding author. 
to develop himself in order to deliver quality up to date care. It is the requirement of General medical council (GMC) to undergo continuous professional development. According to GMC, continuous professional development activities should maintain and improve the quality of care given to patients and the public [1].

The author has found that the most dreadful complication after colorectal operations which involve anastomosis is anastomotic leak. This anastomotic leak increases morbidity as well as mortality. The most challenging aspect of this problem is that there is no definite tool available to predict leak although few modalities are currently used to assess anastomosis. The author strongly feels that there is desperate need to have a definite tool to improve clinical outcome, reduce morbidity and mortality.

Literature search has revealed that reported radiologic leak rate is up to $20 \%$ in lower rectal anastomosis. Leaks cause all sort of complications including paralytic ileus, wound infection, peritonitis, sepsis and abscess formation post operatively. Almost one third of postoperative deaths are the result and consequence of leakage related complications in colorectal surgery [2].

Sadly colorectal anastomosis sometimes leaks in spite of the top efforts of the surgeon. The author has found out that the leakage causes are multi-factorial. Although the two crucial factors are tension in two ends of bowel and inadequate blood flow causing decreased oxygenation at an anastomotic site of the bowel [3]. Unfortunately the presence of these two factors nearly always leads to anastomotic leak.

There is no accurate intra-operative tool to predict an anastomotic leak. Since the arrival of laparoscopy, it has become more challenging to assess anastomosis due to loss of 3 dimensional views and tactile sensation. An absence of true predictor has led to practice of creating de-functioning stoma which is simply a temporary bag for anastomosis in order to minimise mortality and morbidity associated with a leak. It means that these patients require another operation in the future with the aim to reverse this above mentioned stoma.

\section{Introduction}

The author has discovered that it is necessary to facilitate future specialist developments to improve patient care. He was able to act as active leader and effective manager. He also learnt that "Leadership is about hearts and minds and management is about nuts and bolts" [4]. The author became as described by Dr Steven Covey [5] and was able to perform a comprehensive literature search to find a solution for previously stated anastomotic leak problem.

The author proposes that narrow band imaging (NBI) colonoscopy should be used to assess anastomosis and this is the real solution to the problem. Past studies have revealed that intra-operative ordinary colonoscopy can be safely performed to assess anastomosis. This helps mainly detecting anastomotic defects and bleeding. Also past studies has uncovered that intra-operative evaluation of anastomosis with the ordinary colonoscope prevents early anastomotic related problems and eventually reduces anastomotic leak rate [6]. The vital factor for healing in anastomosis is intraluminal vascularity. It is not possible to evaluate intraluminal vascularity with the use of an ordinary colonoscope.

Therefore this problem need to be resolved with the help of some specialised colonoscope and the author believes that narrow band imaging (NBI) colonoscopy is the valid existent solution. Addition of NBI for this purpose is aided property to ordinary colonoscope but as it is only a change in images, it does not put the patient at any extra risk and it is extremely safe.

Narrow Band Imaging (NBI) is a relatively new real-time imaging technique which is an integral component of many modern colonoscopes (Figure 1). This is widely used in adenoma detection but not used at all to assess anastomosis. Narrow Band Imaging (NBI) is a visual image improvement in technology that enhances the visibility of capillaries and other minute structures on the mucosal surface by engaging the light absorption features of haemoglobin at a particular wave length.

The author has found out that the improved visibility made possible by NBI is much easier to handle. We can switch between regular and NBI images at the touch of a button and as often as necessary.

This visual phenomenon achieved from NBI colonsocopy has been used to aid in the detection of early cancers of the lower GI tract and also of the upper GI tract, larynx and bladder [8].

A comparative study revealed that NBI achieved better visualization of the colonic mucosal vascular network than conventional endoscopy [9]. Hence, NBI colonoscopy is assumed to be far more superior in detecting blood flow and vascularity of the large bowel lesions as compared to ordinary white light colonoscopy. Therefore the author proposes that NBI should also be used in assessing anastomosis at the time of operation. 
Our standard current practice is to perform air leak test and to check doughnuts during the operation in order to assess anastomosis. Air leak test is also called Jacuzzi test and that is helpful to identify intra operative leak. That is actually per anus insufflations of air via a syringe while holding the bowel proximal to anastomosis under water. Any leak is obvious with the show of bubbles. Previous air leak testing data indicate a high rate of air leaks as compared to clinical leak of left-sided colorectal anastomosis [10]. So air leak test can give false positive result which is a real problem. Furthermore it is not possible to visualize the vascularity with air leak test and also not possible to identify exact leak site. Therefore it is not routinely possible to correct anastomosis at the time of surgery on the basis of already existing anastomosis checking modalities and these patients usually require de-functioning stoma bag.

It is also our standard existing practice to always check proximal and distal doughnut (2 small portions of bowel), which are attained soon after anastomosis is constructed. The completeness of the doughnut also ensure satisfactory anastomosis but not the confirmation.

Hence by doing endoscopic examination with NBI after construction of anastomosis will give us opportunity to do leak test as usual, to look at an anastomotic integrity and patency of lumen and any visual defects and most importantly allows us to look at the vascularity of mucosa with NBI and compare it with standard technique.

\section{Methodology}

During this learning exercise, it became obvious to the author that good leadership and management qualities are imperative to have in order to develop a new project. He learnt that "managers are people who do things right and leaders are people who do the right things" [11]. The author also realised that it is essential for a successful leader to deliver the following roles according to The Skills/ Will matrix (Figure 2).

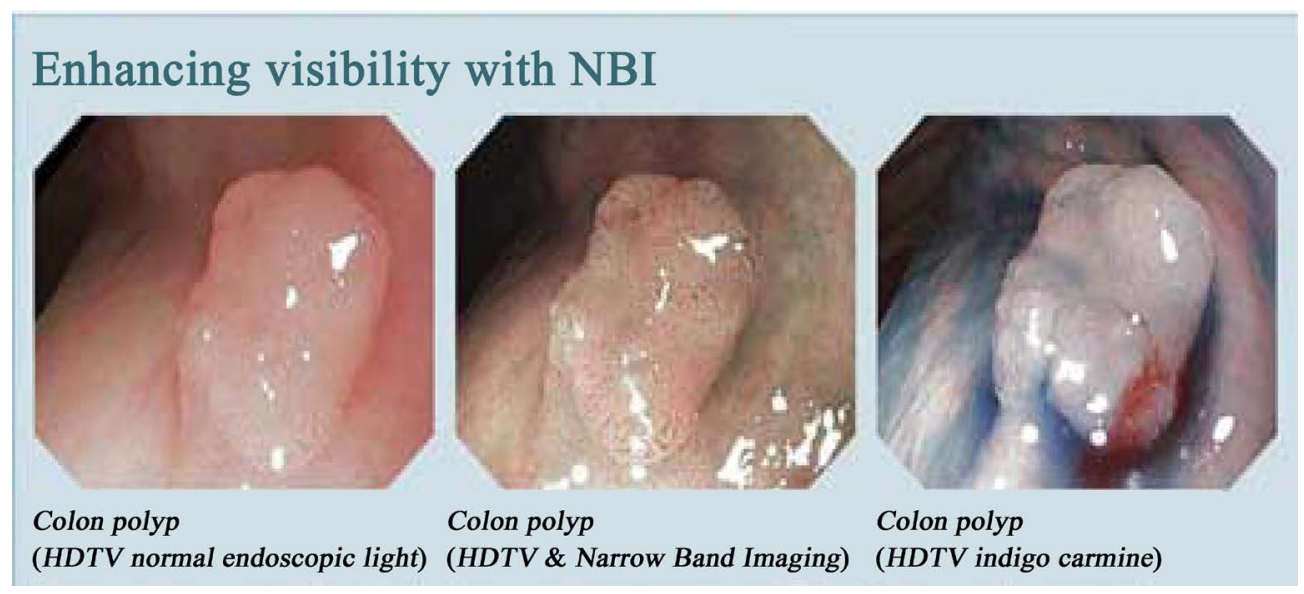

Figure 1. Olympus, no date [7].

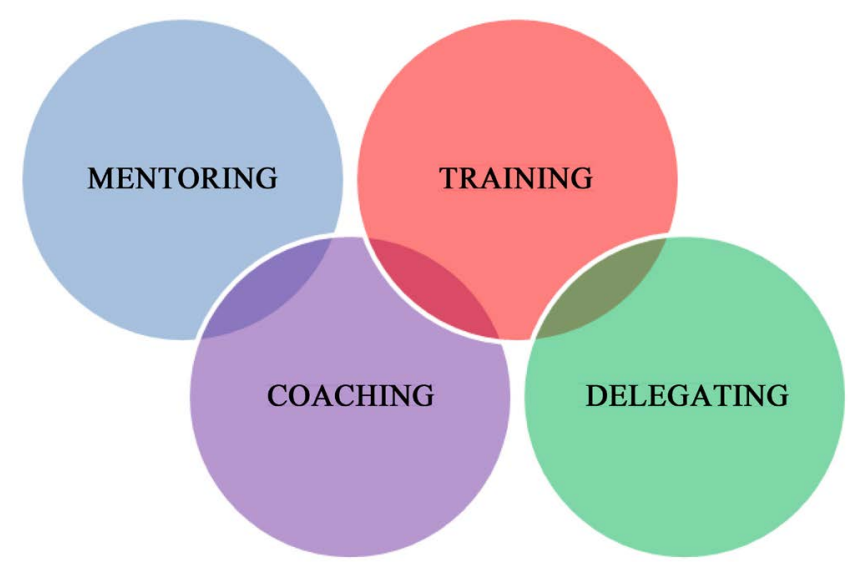

Figure 2. Leader roles. 
He was able to develop methodology and related documents including patient and GP information sheet on the basis of knowledge achieved from literature search demonstrated in the past sections. These documents are imperative and crucial documents for success and ethical approval of a research project. The below mentioned process demonstrates the author's personal responsibility, decision-making and self-direction in designing and executing a response to the need for specialist developments

The author proposes that intra operative Narrow band imaging colonoscopy should be routinely used and is valuable in left sided colonic anastomosis assessment. The author used and learnt PICO model [12] to develop research question for this assignment work.

\section{P (POPULATION)}

All Patients requiring colorectal operation involving left sided anastomosis

\section{I (INTERVENTION)}

Narrow band imaging colonoscopy to assess anastomosis

\section{C (COMPARISON)}

Standard Techniques (Air Leak test + Doughnuts examination) to assess anastomosis

\section{O (OUTCOME)}

Anastomotic leak

The PICO model was found extremely helpful in constructing the foundation of this proposed research study in the author's experience. Further methodology was developed on the basis of this PICO model. The author proposes that the study should be:

- A pilot, single blind, prospective, randomised controlled trial

- Anastomosis will be performed by one experienced colorectal surgeon

- Aim is to include both laparoscopic and open procedures

Inclusion Criteria

All colorectal operation requiring left sided anastomosis:

- Cancer

- Diverticular disease

- Colostomy reversal

\section{Exclusion criteria}

Colorectal operation not requiring left sided anastomosis

Patients not willing to participate

\section{Sample size}

This study will require 30 patients in total divided in 2 groups. We aim to recruit 35 patients to cover any potential future drop out of patient.

\section{Recruitment and Randomisation}

It is also vital to eliminate selection bias therefor Patients will be randomly assigned to one of the two following groups:

\begin{tabular}{cc}
\hline Group & Description \\
\hline Group 1 (Treatment) & Intra-operative NBI Colonoscopy \\
Group 2 (Control) & Air Leak Test + Doughnuts Examination \\
\hline
\end{tabular}

\section{Blinding}

The author also discovered during the development of this portfolio that blinding is crucial in any study to avoid performance bias. It is ideal to have double blinded study which means both patient as well as researcher are blinded to intervention. It was not possible for researcher to be blinded in this study due to obvious reason as they will be performing the procedure; therefore only single blinded study is proposed in which patients will be blinded.

\section{Team working}

The author found out the importance of team working during the development of procedure. He realised that without an effective team, it is not possible to accomplish this research project. The key members of his team consist of his supervisor, local research committee members, colleagues, statistician and most importantly participants. The author learnt that every team goes through different developmental stages as described by below mentioned Tuckman model (Figure 3).

The ideal stage is performing stage where team leader only supervise the rest of the team and it is the respon- 


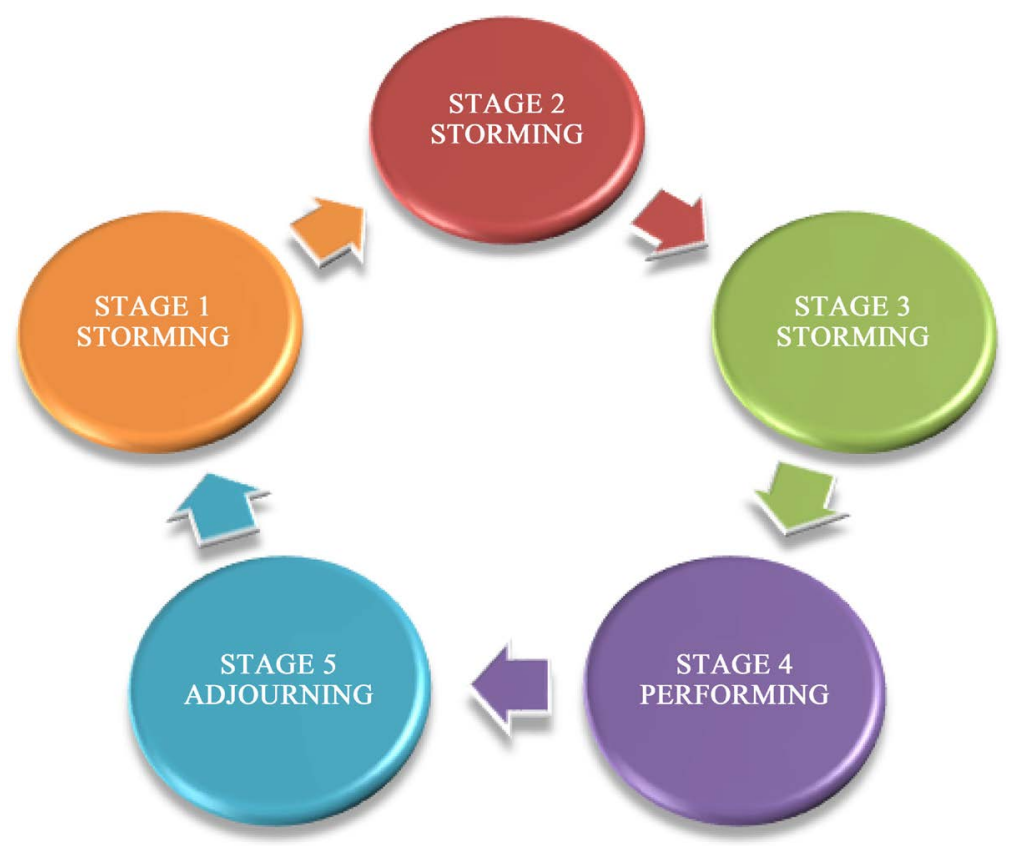

Figure 3. Tuckman’s teams stages.

sibility of a leader to stop his team to go to adjourning stage which is a destructive stage [13]. The author was able to devise a comprehensive procedure with the help of an effective team.

\subsection{Procedure}

1) Patients will be recruited from the colorectal outpatient clinic. This is usually a few days prior to operation. That will ensure that patients are given enough time to understand the study.

2) Once a patient has agreed to participate in the study, they will be screened by Research Team member as to whether they are potential candidates for our study. This will be done against the exclusion/inclusion criteria given. If a patient is a potential trial entrant then an information sheet about the study and an explanation will be provided by Team. The patient will then be allowed to take the sheet home and given time to read through the sheet and decide whether they wish to participate or not. One day before operation or on the day of surgery, the patient will be approached as to whether they will consent for the study or not. If they decide not to take part then operation will be performed in the standard manner and the patient excluded from the study.

3) Once consented patients are randomised by Research Team into one of the TWO (See enclosed flow chart). Randomisation will be performed using a system of sealed envelopes to assign patients into one of the two study groups as shown above.

4) All participating patient's details will be held in a database which is kept in the colorectal surgery department. The database will only hold unit number as a patient identifier and will be password protected.

5) Operation, NBI colonoscopy and Leak test will be performed by the experienced colorectal surgeon.

6) Colonoscopy will be performed using standard endoscopes having both white light and blue/green light functions.

7) Colorectal assessment with NBI colonoscopy will be done in four quadrants proximal and distal to anastomosis ring and images will be collected.

8) Strategy in case air leak test or NBI colonoscopy test reveals a problem will be the clinical judgement of the consultant. The potential options could be:

- De-functioning stoma

- Stitching of the defect

- Redo anastomosis

9) Although we are not expecting any adverse event, any adverse event will be reported and recorded fully and accurately. 
10) All Participating patients will be followed up in clinics in 6 - 8 week time and thereafter as required. Patients will be requested to complete patient satisfaction survey in their first clinic follow up appointment.

\section{FLOWCHART}

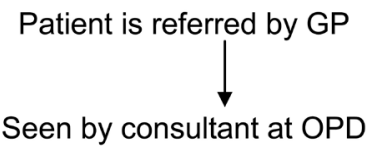

Cancer Patient discussed in

MDT

\section{Decision to operate}
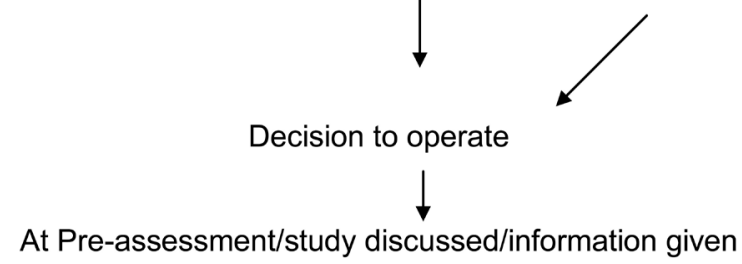

Accepted Participation

\section{Randomisation performed}

Group 1 = Intra-operative Narrow Band Imaging colonoscopy

Group 2 = standard anastomosis assessment (Intra operative Air leak test and confirming completeness of the 2 doughnut's)

Assessment as per randomisation Follow up as per protocol / routine Satisfactory survey at 6 week follow up appointment

\section{Declined participation}

Routine care as per Consultant care pathway Follow up as routine

\subsection{Consent}

Consent is an agreement to receive a treatment or intervention between a patient and health care individual proposing the treatment. The author has learnt during the development of sample consent form that it is paramount for any consent to have three vital components (Figure 4).

According to above model, consent should always be voluntary without any influence. It should be fully informed decision with the provision of all the possible options to patients. Patient should have capacity to make a decision for him and in case of absent capacity; decision should be made in his best interest. These are the fundamental principle of all the consent procedures. The author also suggests having the same consent model for his research participants.

\subsection{Communication}

The author also learnt that its paramount to maintain communication at all levels at all times and was able to 


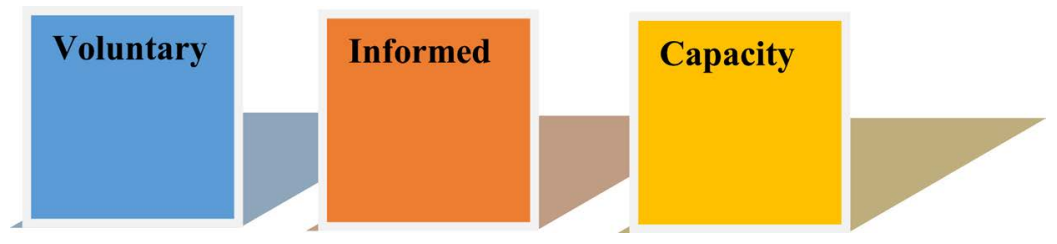

Figure 4. Consent model [14].

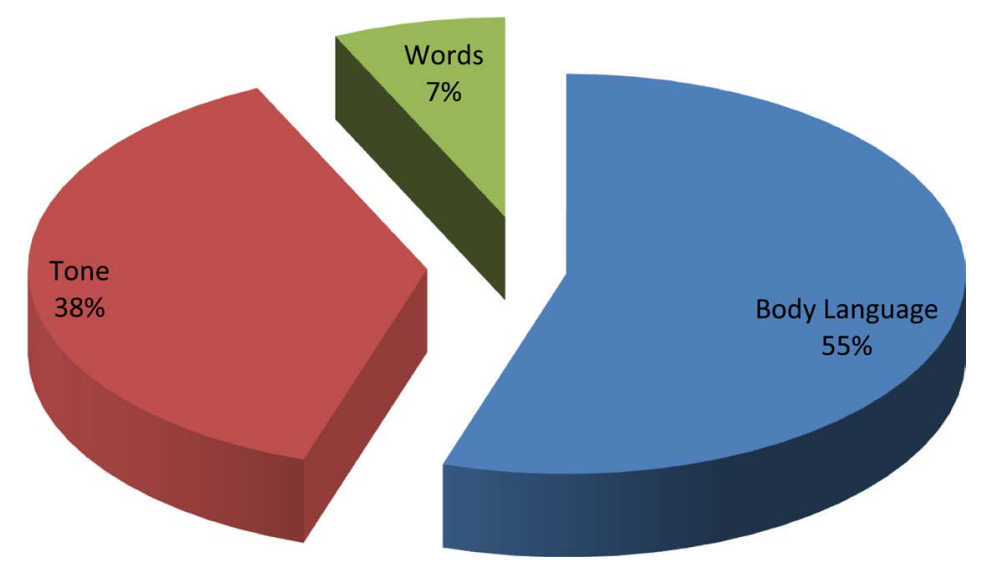

Figure 5. Mehrabian communication chart.

develop his communication skills during this learning process. He found out that most important aspect in communication is body language [15] but written information also plays significant impact (Figure 5).

Therefore the author was able to develop a sample information letter for GP as well as patient. He also learnt that communication need for GP and patient is different. It is crucial to use the appropriate language and tone for a medical colleague as well as for a patient for a successful communication.

\subsection{Sample GP Letter}

\section{Dear Dr}

Your patient .DOB

is under treatment in the Colorectal Unit. In due course of his/her treatment will require Surgical Intervention needing Left sided colonic anastomosis.

We are currently conducting a research study to check anastomotic integrity. Your patient has consented to participate in the research study and will be randomised between the 2 groups.

Group 1. Direct visualisation of anastomosis with Narrow Band Imaging colonoscopy

Group 2. Standard anastomosis and usual routine assessment with Intra operative Air leak test and confirming completeness of the 2 doughnuts.

To ensure blinding of the study we will not reveal the treatment arm. However, if you have any concerns at all about your patient in regard to this surgical intervention, or you have any specific queries relating to this study please contact one of the research team at Hospital.

Yours sincerely

Research Team 


\subsection{Sample Participant Information Sheet}

Explain the idea behind this study? Bowel anastomosis is a surgical procedure that is performed to join two ends of bowel together inside the body after removing diseased part of the bowel. An anastomotic leak is a breakdown along this join which causes waste material to leak inside the body. This type of complication leads to increased mortality and morbidity.

When a surgeon performs a join, care is taken at every step of the procedure. Once the surgeon is satisfied that the join is complete, it is tested for leak ensuring that it is air tight and the two cut ends of the bowel are visually checked for completion.

We can also perform a visual inspection of join by using a colonoscope camera. We want to use a specialized colonoscope called Narrow Band Imaging (NBI) colonoscope to inspect the join. It is better technology and allows check for healthy blood supply.

We wish to assess effectiveness of NBI Colonoscopy in join inspection. The only way of doing this is to divide patients into two groups. One group will be assessed with NBI colonoscopy as well as standard testing technique. The other group will only be accessed by using standard join testing technique. To make it fair, the patients will be randomly allocated by a computer.

\section{Where is the research being conducted?}

The research is being conducted at Hospital.

\section{Why have I been chosen?}

We are requesting all patients who requires a potential anastomosis whether they would like to participate or not. You will be excluded from this study if you do not require a join.

\section{What will I be asked to do?}

The operation will be performed as normal under a general anaesthetic. You will be randomly allocated to one group. We will be taking a few photographs of your bowel and maybe internal organs. You will not be aware about the type of technique used in your operation.

All our patients will be reviewed after six weeks in OPD clinic to check progress and if necessary arrangements will be made to review early. You are expected to inform us of any untoward affects you may have related to your surgery.

\section{Do I have to participate?}

It is your decision whether to participate or not. If you do decide to take part, you will be requested to sign a consent form. You are able to change your mind without providing a justification at any time.

If you decide not to take part in the study it will not change your operation or treatment right. If you leave the study at any time, your care will not be compromised at all. You will also be seen in the outpatient clinic after six weeks to check on your progress.

Who will perform my operation?

There will be no difference in the surgeon who performs your operation. This surgeon will be Colorectal Consultant or a senior member of his surgical team. The surgeon who operates on you will be familiar with performing the standard and new join checking techniques.

\section{Is there any possible benefit to me if I take part?}

If you are allocated to receive the NBI Colonoscopy as well, we would anticipate that we can assess join more precisely and deal with any join related problems earlier than the standard operation. Moreover there is potential chance of avoiding a temporary stoma bag. It is our assumption that this study will hopefully show this benefit.

\section{Are there any risks, disadvantages or costs in taking part?}

NO. The operation is identical to the standard operation except for the use of an Intra-operative NBI colonoscopy. The Colonoscopy has been safely used In the past for checking joins without any increase risk. It will only increase operating time by 15 minutes.

The time you wait for your operation or the surgeon performing the operation is not effected by taking part in this study. No extra hospital visits are required so you will not incur any financial cost in participating.

\section{Will the information about me be kept confidential?}

YES. All information will be stored electronically and entries will be coded so that they do not refer to you directly. It is part of our job to make sure that all the information is safe and confidential. Such information will only be seen by the doctors and nurses looking after you.

We will request for your permission to inform your GP (family doctor) that you are participating in this study, but he/she will not have access to the information from your records without your agreement.

\section{What will happen to the information about me after the study?}

Once the study is completed, it is likely that the results will be published in a medical journal. However, you would not be identifiable as an individual in any of these publications. We can provide a copy of the results of this study for you upon completion at your request.

You can see the information that we have about you at any time by contacting any of the following named below.

Thank you

Please contact the research team

In case you need any more information 


\subsection{Significance}

This area of research is particularly important for the following reasons:

1) It will significantly reduce anastomotic leak rate. With the ability to predict anastomotic leak at the time of surgery, it would enable surgeon to correct that and hence reduce leak rate.

2) As this procedure will give surgeons more confidence in the integrity of anastomosis, it will decrease the need of de-functioning stoma hence patient is less prone to stoma related problems and avoidance of second operation requiring stoma reversal.

3) This method will also detect other anastomosis related problems including stricture and bleeding therefore lead to their intra-operative correction and significant post op reduction of these complication.

4) Ultimately this will decrease average hospital stay of patients requiring left sided anastomosis.

5) There will be significant cost savings to the trust by minimising hospital stay with the avoidance of anastomotic associated complications and second operation required to reverse stoma or correct complications of anastomosis.

6) Improve survival by reducing morbidity and mortality rate.

\subsection{Ethical Consideration}

It is our duty as doctors to provide excellent care and try to reduce anastomotic related problems, associated with morbidity and mortality. All patients approached to participate in this study will be explicitly requested for their consent in using their data. All parts of the study will be explained, including the potential benefits of the study to them. It will also be explained that non-participation will not affect in any way the treatment that they will receive.

All data collected will be anonymised at source so that privacy of patient information is not put at risk. There is no need to collect any patient identifiable information, therefore id numbers will be used at all stages in the process. It is recognised that this study is only single blinded due to nature of procedure as surgeon will always be aware of used technique to assess anastomosis.

The research proposed here has been deliberately kept as a pilot study at this level as it is not possible for single centre to have large enough number of patients requiring left sided anastomosis in required time scale. It is however hoped that the proposed research will help to assess anastomotic integrity. It will also provide base and set standard and guidelines for potential national multicentre trial.

\section{Summary}

It is vital for Health care individuals to be able to recognize and appraise current opportunities and challenges, and develop for the future. This is a unique exiting opportunity for the author to learn and develop himself.

NBI colonoscopy is not routinely used to assess anastomosis vascularity and defect. The author proposes that frequent use of NBI colonoscopy will lead to significant reduction in anastomotic leak and stricture rate, by assessing vascularity of both proximal and distal end and direct visualization and recognition of any defect. The author is able to produce a well-structured research project to prove this hypothesis.

SOAR model suggested by Kumar [16] was used by the author during this developmental learning process. He was able to set realistic goals which ultimately helped him in formulating a structured plan to achieve these aims (Figure 6).

The author came to know that it was vital to have self-motivation first as mentioned above and then combination of right opportunities and appropriate abilities could lead to fruitful required outcome.

He also used GROW model created by Sir John Whitmore [17] to acquire new knowledge (Figure 7).

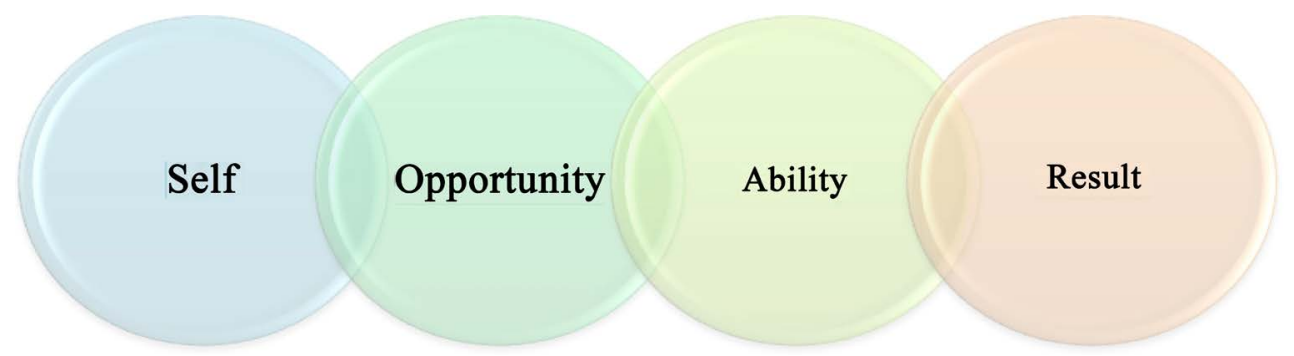

Figure 6. SOAR model. 


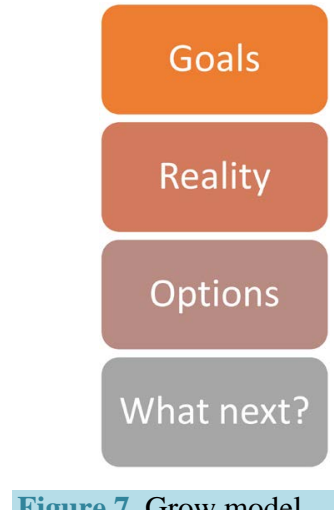

Figure 7. Grow model.

The author was able to set realistic goals and then worked hard by utilizing his all available options which ultimately resulted in successful outcome in the form of a well-structured research project which was now ready to go to next level after ethics committee permission.

\section{References}

[1] GMC (2012) Continuing Professional Development. http://www.gmc-uk.org/Continuing_professional_development_guidance_for_all_doctors_1114.pdf_56438625.pdf

[2] Alberts, J.C.J., Parvaiz, A. and Moran, B.J. (2003) Predicting Risk and Diminishing the Consequences of Anastomotic Dehiscence Following Rectal Resection. Colorectal Disease, 5, 478-482. http://dx.doi.org/10.1046/j.1463-1318.2003.00515.x

[3] Kingham, T.P. and Pachter, H.L. (2009) Colonic Anastomotic Leak: Risk Factors, Diagnosis, and Treatment. Journal of the American College of Surgeons, 208, 269-278. http://dx.doi.org/10.1016/j.jamcollsurg.2008.10.015

[4] Blanchard, K.H., Zigarmi, P. and Zigarmi, D. (2013) Leadership and the One Minute Manager: Increasing Effectiveness through Situational Leadership. William Morrow, an Imprint of HarperCollins, New York.

[5] Covey, S.R. (1989) The 7 Habits of Highly Effective People: Powerful Lessons in Personal Change. Free Press, Location.

[6] Ishihara, S., Watanabe, T. and Nagawa, H. (2008) Intraoperative Colonoscopy for Stapled Anastomosis in Colorectal Surgery. Surgery Today, 38, 1063-1065. http://dx.doi.org/10.1007/s00595-007-3740-0

[7] Olympus [No Date] Narrow Band Imaging (NBI), a New Wave of Diagnostic Possibilities. http://www.olympus.cz/medical/en/medical_systems/applications/gastroenterology_1/narrow_band_imaging_nbi_in _gastroenterology/narrow_band_imaging_nbi_2.jsp

[8] Mannath, J., Subramanian, V., Hawkey, C.J. and Ragunath, K. (2010) Narrow Band Imaging for Characterization of High Grade Dysplasia and Specialized intestinal Metaplasia in Barrett’s Esophagus: A Meta-Analysis. Endoscopy, 42, 351-359. http://dx.doi.org/10.1055/s-0029-1243949

[9] Chiu, H.M., Chang, C.Y., Chen, C.C., Lee, Y.C., Wu, M.S., Lin, J.T. and Wang, H.P. (2007) A Prospective Comparative Study of Narrow-Band Imaging, Chromoendoscopy, and Conventional Colonoscopy in the Diagnosis of Colorectal Neoplasia. Gut, 56, 373-379. http://dx.doi.org/10.1136/gut.2006.099614

[10] Hall, J.F., Marcello, P.W., Read, T.E., Ricciardi, R., Roberts, P.L. and Schoetz, D.J. (2009) Anastomotic Leak Testing after Colorectal Resection: What Are the Data? Department of Colon and Rectal Surgery, PubMed, Massachusetts.

[11] Nannus, B. and Bennis, W.G. (2007) Leaders: Strategies for Taking Charge. 2nd Edition, Harper Business. Collins Business Essentials.

[12] Booth, A. and Brice, A. (2004) Evidence Based Practice for Information Professionals: A Handbook. Facet Publishing, London, 61-70.

[13] Tuckman, B.W. and Jensen, M.A.C. (1977) Stages of Small Group Development. 2nd Edition.

[14] NHS (2014) Consent to Treatment. http://www.nhs.uk/conditions/Consent-to-treatment/Pages/Introduction.aspx

[15] Mehrabian, A. (1970) Tactics of Social Influence.

[16] Kumar, A. (2007) Personal, Academic and Career Development in Higher Education SOARing to Success. Routledge.

[17] Whitmore, J. (2009) Coaching for Performance: GROWing Human Potential and Purpose-The Principles and Practice of Coaching and Leadership. 4th Edition, Icholas Brealey Publishing. 